\title{
Efficacy of contrast-enhanced ultrasonography and shear-wave elastography in conjunction with BI- RADS classification for diagnosis of small breast nodules
}

\author{
Yan Shen \\ Gongli Hospital \\ Jie He \\ Gongli hospital \\ Miao Liu \\ Gongli hospital \\ Jiaojiao Hu \\ Gongli Hospital \\ Yonglin Wan \\ Gongli Hospital \\ Tingting Zhang \\ Gongli Hospital \\ Jun Ding \\ Gongli Hospital \\ Jiangnan Dong \\ Gongli Hospital \\ Xiaohong Fu ( $\square$ fuxiaohong66@126.com )
}

Research article

Keywords: Contrast-enhanced ultrasound, Shear-wave elastography, Small breast nodule, Ultrasound

Posted Date: August 27th, 2020

DOI: https://doi.org/10.21203/rs.3.rs-36646/v2

License: (9) This work is licensed under a Creative Commons Attribution 4.0 International License.

Read Full License 


\section{Abstract}

Background: Identification of malignancy in small breast nodules can be difficult using conventional methods, especially in patients with dense breast tissue. Advanced imaging techniques, including contrast-enhanced ultrasound (CEUS) and shear-wave elastography (SWE), could be used in conjunction with Breast Imaging Reporting and Data System (BI-RADS) classification to characterize these nodules more effectively. This study aimed to evaluate the use of CEUS and SWE for the differentiation of benign from malignant small $(\leq 2 \mathrm{~cm})$ breast nodules.

Methods. This retrospective study reviewed the medical records and imaging data of 302 patients who underwent evaluation for 305 small breast nodules from November 2015 to December 2019. BI-RADS classification of nodules and the results of CEUS and SWE were retrospectively analyzed; the diagnostic efficacy of these techniques was evaluated by comparison with pathology results. Receiver operating characteristic curves were analyzed based on the CEUS patterns and shear-wave velocity values of nodules. The sensitivities, specificities, positive and negative predictive values, and accuracies of BIRADS, CEUS, SWE, and a combination of all three methods for identifying benign versus malignant small breast nodules were investigated.

Results. CEUS was effective at diagnosing malignant nodules when at least two out of nine suspicious features were present. Receiver operating curve analysis revealed that the best cut-off value for SWE was $3.7 \mathrm{~m} / \mathrm{s}$. For the diagnosis of benign breast nodules, the BI-RADS classification was reduced by one level when both CEUS and SWE were used, and was unchanged when either CEUS or SWE alone were used; the highest and lowest levels were category 5 and 3 , respectively. Furthermore, when using the combined method, $75.8 \%(91 / 120)$ of small breast nodules with a BI-RADS category 4 A classification avoided the need for coarse needle biopsies.

Conclusion: Both CEUS and SWE can be used as auxiliary methods for clarifying BI-RADS classification of breast nodules, and a combination of these techniques may provide more diagnostic efficacy for identifying malignancy in small breast nodules.

\section{Background}

Breast cancer has the second highest tumor-related mortality rate in women [1], and its morbidity is increasing, especially in patients with early-onset disease. There is a consensus that the recognition and treatment of early-stage breast cancer can reduce mortality [2]. However, small breast nodules lack the typical clinical characteristics of breast cancer and are easily missed by clinicians; some small malignant breast nodules (diameter $\leqq 2 \mathrm{~cm}$ ) present similar ultrasonographic features with benign nodules.

Ultrasound imaging is important for screening patients for breast cancer. In recent years, the broad use of the Breast Imaging Reporting and Data System (BI-RADS) [3] for conventional ultrasonography has improved the predictive value of breast cancer diagnosis. The BI-RADS is primarily based on a subjective judgment by clinicians; thus, conventional ultrasound diagnosis remains imperfect [4-5]. As a result, 
ultrasound-guided breast nodule biopsy must be performed in the diagnosis of early breast cancer. According to management guidelines, nodules that meet the criteria for BI-RADS category 4A are suspected to be malignant in $3 \%$ to $10 \%$ of cases, and a biopsy is typically recommended in these patients [6]. However, biopsies are invasive, with underlying risks associated with needle use and increased financial and psychological burden for patients. Therefore, the need for biopsies in patients with low-risk nodules remains controversial.

More advanced technologies have recently been introduced that may improve the accuracy of breast nodule diagnosis, including contrast-enhanced ultrasound (CEUS) and shear-wave elastography (SWE). CEUS is a blood pool imaging technique in which a gaseous microbubble of contrast agent with a diameter similar to a red blood cell is injected into a surrounding vein. CEUS obtains a large contrast sonogram by leveraging the strong gas-scattering properties and differing acoustic characteristics of human tissues to increase differences in the acoustic impedances of circulating blood or lesions and adjacent tissues [7]; the resulting images can provide information regarding the morphology and distribution of blood vessels associated with tumors. SWE can facilitate the differentiation of benign from malignant lesions by measuring the propagation velocity of shear waves in tissue to determine the tissue hardness.

Previous studies have confirmed that multi-mode ultrasound (US combined with CEUS, SWE) can significantly improve the diagnostic efficacy of breast cancer [8], and some studies have confirmed that CEUS combined with SWE can greatly improve the detection rate of small breast cancer [9]. However, few studies have evaluated the ability of US combined with CEUS and SWE in the diagnosis of small breast cancer and its value in terms of avoiding unnecessary biopsy. The purpose of this study was to evaluate the diagnostic efficacy of CEUS and SWE for identifying malignant small breast nodules to guide further refinement of the BI-RADS classification system and to provide better management of individual cases.

\section{Methods}

\section{Ethical statement}

This study was carried out in accordance with the Declaration of Helsinki, and written informed consent was obtained from all patients. The protocol was approved by the Ethics Committee of Gong Li Hospital. The need for written informed consent from patients was waived due to the retrospective nature of this study.

\section{Patients}

From November 2015 to December 2019, 676 patients with breast nodules underwent conventional ultrasound, CEUS, and SWE at our institution. Patients were eligible for inclusion in this study if they had: 
(a) a breast nodule with a diameter of $\leq 2 \mathrm{~cm}$; (b) no prior treatment for breast nodule before ultrasonography; (c) pathological results obtained from surgical or needle biopsies; and (d) a solid or mostly solid breast nodule (cystic portion of less than $25 \%$ of the total volume). Patients were excluded from this study if they: (a) were pregnant or lactating or (b) had only low-quality breast nodule images that were too poor for analysis.

\section{Imaging instruments}

A Philips iU Elite and EPIQ 5 Ultrasound diagnostic apparatus equipped with CEUS software was used for CEUS imaging (Philips Medical Systems, Bothell, WA, USA). The apparatus was equipped with a L12-5 linear probe and operated at a 5 to $12 \mathrm{MHz}$ frequency range. A Siemens Acuson S3000 Ultrasound diagnostic apparatus (Siemens Medical Solutions, Mountain View, CA, USA) equipped with SWE imaging software was used for SWE imaging. It was equipped with a $9 L 4$ linear probe and operated at a 4 to 9 $\mathrm{MHz}$ frequency range.

\section{Imaging techniques and analysis}

For ultrasonography, each patient was placed in a supine position with their hands raised above their head. To detect the lateral breast, the patient was turned onto their side, with both breasts fully exposed. Parameters such as gain and depth were adjusted according to the position and size of the breast mass. Operators recorded information about nodular features, including location, size, shape, orientation, margin, echo pattern, posterior acoustic features, calcification, and vascularity; the lesion was then categorized based on the BI-RADS classification system. For patients with multiple nodules, the BI-RADS classification was based on the nodule in the highest category.

Subsequently, SWE, which assesses quality, velocity, and image retention, was performed; nodules were imaged where they were most clear. After identifying the maximum longitudinal section, selected nodules were imaged within the sampling frame. The size was adjusted at least two times, and the patient was instructed to hold their breath for a few seconds after image stabilization. The quality of the images obtained after assessment of shear-wave elasticity distributions was graded from high to low based on a color-coded system ranging from green to yellow to red. Images with a uniform distribution and a regional green area were defined as good quality; otherwise, images were considered to be of poor quality.

After the identification of high-quality images, shear-wave velocity (SWV) was measured to determine the tissue stiffness. The SWE speed mode was used to obtain SWV values directly within the twodimensional space distribution of the SWE imaging map, with the SWV displayed as either red, yellow, green, or blue to depict speeds ranging from fast to slow. The SWV range was gradually adjusted (maximum of $10.0 \mathrm{~m} / \mathrm{s}$ ) when the internal regions of the nodules were red or yellow with surrounding blue or green. Multiple (generally 5-7) regions of interest (ROIs) were positioned within different areas 
inside the nodules (upper, lower, middle, and periphery, at the highest and lowest speeds). SWV measurements were recorded in the effective measurement areas, and average SWV values $(\mathrm{m} / \mathrm{s})$ for each nodule were obtained.

For CEUS imaging, sections with rich blood flow, large blood vessels, or irregular shapes were selected, and sections with large calcifications accompanied by wide sound shadows were avoided. After switching to breast CEUS mode, dual real-time displays were used to continuously track and observe the two-dimensional images, allowing the radiologist to stabilize the image on the same section. The depth was adjusted so that enough normal breast tissue was present around the nodule for comparison. The focal point was set behind the nodule with a mechanical index of 0.07 . Twenty-five $\mathrm{mg}$ of the contrast agent (SonoVue, Bracco SpA, Milan, Italy) was suspended in $5 \mathrm{~mL}$ of a $0.9 \%$ sodium chloride solution and shaken by hand. The probe was then gently placed against the skin in front of the nodule to avoid applying excessive pressure. A rapid intravenous bolus injection was performed with $4.8 \mathrm{~mL}$ of the contrast agent suspension followed by $5 \mathrm{~mL}$ of the $0.9 \%$ sodium chloride solution. Video recording commenced concurrently with contrast agent injection, and the dynamic perfusion process of the nodule was continuously observed for at least $3 \mathrm{~min}$. During the entire angiography process, the patient was asked to breathe normally and to remain still. The probe was maintained at the same section to avoid human interference.

According to a previously published article [10] and our experience, the following nine variables were evaluated during CEUS examinations: 1) enhancement intensity (low enhancement, equal enhancement, high enhancement); 2) order of enhancement (concentricity, non-concentricity); 3) change (difficult to distinguish, shrinking, unchanged, expanding); 4) enhancement uniformity (uniform, non-uniform); 5) enhancement defects (present or absent); 6) morphology after enhancement (regular, difficult to distinguish, irregular); 7) enhanced posterior boundary (clearly distinguishable, difficult to distinguish, unclear); 8) claw sign (present or absent); and 9) nourishing blood vessels (present or absent). When the evaluations were inconsistent, two ultrasound physicians discussed the features to reach a consensus.

All conventional ultrasound, CEUS, and SWE images were stored on the hard disk drive of the ultrasonic instrument for further analysis. All SWE image acquisition and data analyses were completed by two radiologists ( $\mathrm{JH} \& \mathrm{YW}$ ) with more than 3 and 10 years of experience in SWE and breast ultrasonography, respectively. CEUS images were collected by two radiologists (ML \& TZ) with more than 3 and 10 years of experience in breast CEUS and breast ultrasonography, respectively. CEUS images were evaluated by two radiologists (YS \& JH) with more than 5 and 10 years of experience in CEUS and routine ultrasound examinations, respectively.

\section{Statistical analysis}

Statistical Package for the Social Sciences (SPSS) software (version 22.0, SPSS Inc., Chicago, IL, USA) and MedCalc software (version 19.0.7, Mariakerke, Belgium) were used for statistical analyses. 
Continuous variables were expressed as mean \pm standard deviation or range and compared using the independent-samples $t$-test. Categorical data were expressed as numbers with percentages and compared using the chi-squared test or the Fisher's exact test. Receiver-operating characteristic (ROC) curves were used to analyze the diagnostic value of classifying small breast nodules using each of the four diagnostic methods (BI-RADS, CEUS, SWE, and the combined method). The areas under the curve (AUCs) for the diagnostic ability of the four methods were calculated, and the Cochran $Q$-test and $z$-test were used for statistical analysis. The best cut-off values were obtained from ROC analysis, and the corresponding sensitivities (SEN), specificities (SPE), positive predictive values (PPV), negative predictive values (NPV), and accuracies (ACC) were calculated. Two-sided $P$-values of $<0.05$ were considered statistically significant.

\section{Results}

\section{Pathology}

A total of 302 patients were enrolled in the study. Among these, three patients had two nodules with the same highest BI-RADS classification, thus making a total of 305 enrolled nodules. The average age of the patients was $49.2 \pm 16.4$ years (18-84). Of the 305 small breast nodules assessed, 192 (63.0\%) were determined to be benign based on pathologic examinations, including $83(43.2 \%)$ fibroadenomas, 41 (21.4\%) adenopathies, 39 (20.3\%) adenopathies with fibroadenomas, 17 (8.9\%) intraductal papillomas, nine $(4.7 \%)$ cases of inflammation or abscesses, one $(0.5 \%)$ tubular adenoma, and one $(0.5 \%)$ cavernous hemangioma. The other 113 nodules $(37.0 \%, 113 / 305)$ were determined to be malignant, including 78 (69.0\%) invasive ductal carcinomas, 11 (9.7\%) ductal carcinomas in situ, 11 (9.7\%) papillary carcinomas, five (4.4\%) invasive lobular carcinomas, five (4.4\%) mixed cancers, two $(1.8 \%)$ mucinous carcinomas, and one $(0.9 \%)$ invasive adenocarcinoma.

\section{Ultrasonographic predictors of malignancy}

There were no significant differences in patient age $(t=0.693, P=0.406)$, breast nodule size $(t=2.452, P$ $=0.118)$, or breast nodule location $\left(\chi^{2}=0.759, P=0.226\right)$ between benign and malignant nodules. Compared to benign breast nodules, a higher proportion of malignant nodules had irregular shapes (82.3\%, 93/113; $P \otimes 0.001)$, nonparallel orientations $(60.2 \%, 68 / 113, P \otimes 0.001)$, and distinct boundaries $(83.2 \%, 94 / 113, P \otimes 0.001)$. Other features were also more commonly observed in malignant lesions, including calcification $(46.0 \%, 52 / 113, P \otimes 0.001)$, presence in intramammary ducts $(6.2 \%, 7 / 113)$, internal vascularity $(54.9 \%, 62 / 113, P \otimes 0.001)$, and lymph node metastasis $(11.5 \%, 32 / 113, P \otimes 0.001)$ (Table 1 , Fig. 1). 
There were 25 cases of BI-RADS 3 (benign: 25, 100\%; malignant: 0, 0.0\%), 137 cases of BI-RADS 4A (benign: 120, 87.6\%; malignant: 17, 12.4\%), 78 cases of BI-RADS 4B (benign: $45,57.7 \%$; malignant: 33 , 42.3\%), 60 cases of BI-RADS 4C (benign: 2, 3.3\%; malignant: 58, 96.7\%), and 5 cases of BI-RADS 5 (malignant: $5,100.0 \%)$ ). The cut-off value for the BI-RADS classification system was determined to be category 4B, with a SEN, SPE, PPV, NPV, ACC, and AUC of $82.3 \%, 74.5 \%, 65.5 \%, 87.7 \%, 77.4 \%$, and $78.4 \%$, respectively.

Using CEUS, high enhancement was observed in 188 cases (benign: 108, 57.4\%; malignant: 80, 42.6\%); centripetal enhancement in 99 cases (benign: 28, 28.3\%; malignant: 71,71.7\%); inhomogeneous enhancement in 166 cases (benign: 82, 49.4\%; malignant: 84, 50.6\%); filling defect in 25 cases (benign: 2 , 8.0\%; malignant: 23, 92.0\%); irregular shape after enhancement in 164 cases (benign: 70, 42.7\%; malignant: $94,57.3 \%$ ); volume expansion in 72 cases (benign: $8,11.1 \%$; malignant: $64,88.9 \%$ ); unclear boundary after contrast enhancement in 134 cases (benign: $38,28.4 \%$; malignant: $96,71.6 \%$ ); crab foot sign in 29 cases (benign: 1, 3.4\%; malignant: 28, 96.6\%); and nourishing vessel sign in 57 cases (benign: 8, 14.0\%; malignant: 49, 86.0\%). Malignant nodules could be identified using CEUS when at least two out of nine suspicious malignant signs manifested at the same time, with a SEN, SPE, PPV, NPV, and ACC of $83.2 \%, 87.5 \%, 79.7 \%, 89.8 \%$, and $85.9 \%$, respectively.

The diagnostic efficacy of SWE was determined according to the mean SWV values. The mean SWV was $5.2 \pm 1.6 \mathrm{~m} / \mathrm{s}$ for malignant nodules, which was significantly higher than the mean SWV of $3.1 \pm 1.1 \mathrm{~m} / \mathrm{s}$ for benign nodules $(P \otimes 0.001)$. Based on ROC curve analysis, the cut-off value for SWV was $3.7 \mathrm{~m} / \mathrm{s}$. Among the 131 cases $\geq 3.7 \mathrm{~m} / \mathrm{s}$, $33(25.2 \%)$ were benign and 98 (74.8\%) were malignant. Among the 174 cases $<3.7 \mathrm{~m} / \mathrm{s}, 159$ (91.4\%) were benign and 15 (7.6\%) were malignant. The SEN, SPE, PPV, NPV, ACC, and AUC was $86.7 \%, 82.8 \%, 74.8 \%, 91.4 \%, 84.3 \%$, and $84.8 \%$, respectively.

Using the CEUS and SWE combined method, the BI-RADS classification of the nodules was increased by one category and reduced by one category when diagnosing malignant nodules and benign nodules, respectively. If either CEUS or SWE alone was used to diagnose benign nodules, the BI-RADS classification remained unchanged. The SEN, SPE, PPV, NPV and ACC for the combined method of diagnosis was $88.5 \%, 87.0 \%, 80.0 \%, 92.8 \%$, and $87.5 \%$, respectively.

The Cochran $Q$ test revealed significant differences between the four methods (Cochran $Q=19.573, P<$ 0.01). The AUCs of the ROC curves for the diagnostic efficacy of BI-RADS, CEUS, SWE, and the combined method were $78.4 \%, 85.3 \%, 84.8 \%$ and $87.7 \%$, respectively, with the combined diagnostic method having a significantly higher AUC ( $z$-value 4.553, $P<0.01$; Tables 2,3 ; Fig. 2$)$.

\section{Discussion}

Imaging examinations, especially molybdenum target radiography, magnetic resonance imaging, and ultrasonography play an important role in the early screening and diagnosis of breast diseases. Over the 
past three years, studies outside of China have suggested that large-scale breast cancer screening programs can reduce overall breast cancer mortality by $20 \%$ and early breast cancer mortality by $60 \%$ [11]. However, in comparison to women of other ethnicities, Chinese women generally have relatively smaller breast volumes with higher proportions of dense breast tissue; these characteristics can reduce the sensitivity of mammography for identifying malignant breast lesions. In these cases, ultrasound is a more effective method for characterizing suspicious lesions [12].

Conventional ultrasound can clearly show a variety of breast lesion characteristics, including location, number, size, shape, orientation, perimeter, internal echo, rear features, calcification, blood supply, and associated axillary lymph nodes. In clinical practice, however, two-dimensional ultrasound manifestations of benign and malignant nodules sometimes share overlapping characteristics, especially in patients with small breast tumors. The BI-RADS classification system, which standardizes breast ultrasonography reporting worldwide [13], has been shown to improve the sensitivity of identifying malignant masses; however, the false-positive rate remains high [14].

Based on years of clinical experience, we believe that the biological characteristics of small breast cancers are less commonly manifested and that signs of malignancy in these small tumors are more difficult to observe using two-dimensional ultrasound imaging. Even color Doppler imaging cannot adequately detect obvious differences in blood flow for benign nodules, making differential diagnosis even more difficult. In addition, the pathological characteristics of breast cancer tissues are diverse, which can lead to both false positives and false negatives. For example, some in situ cancers with small and atypical nodules do not display hyperechoic halos or burr signs. Moreover, some small invasive ductal carcinomas, mucinous carcinomas, and papillary carcinomas manifest with clear boundaries, few lobes, and parallel growth, leading to false negatives. Finally, some sclerosing adenoses, intraductal papillomas with inflammation, and other benign lesions exhibit obscure boundaries, leading to false infiltration and false positives. Therefore, the use of conventional ultrasonography, which relies on the recognition of distinctive morphological features, can be problematic during the evaluation of small breast nodules.

In addition to morphological features, other tumor characteristics have been evaluated for their applicability to imaging-based diagnoses. For example, previous studies have shown that angiogenesis plays an important role in tumor growth and metastasis [15]. Tumor blood flow has been evaluated using Doppler ultrasound with some success; however, this modality can only identify a blood flow signal above the threshold of large vessels and the wall filter [16]. On the other hand, real-time CEUS can provide more accurate information about the morphology and distribution of the blood vessels associated with tumors [15]. Moreover, SWE can quantitatively evaluate the hardness of breast nodules $[17,18]$. Therefore, conventional ultrasound combined with CEUS and SWE techniques can comprehensively evaluate nodules based on the shape, micro-blood flow, and hardness of nodules to improve diagnostic efficacy.

CEUS is an advanced technique for microperfusion imaging using a safe contrast medium. It can dynamically display the microperfusion of tumors, lymph nodes, and surrounding tissues in real-time, and it plays significant roles in the diagnosis and targeted therapy of breast diseases, detection of metastatic 
sentinel lymph nodes, and evaluation of neoadjuvant chemotherapy efficacy. CEUS can qualitatively characterize benign and malignant lesions by identifying the homogeneous and centripetal enhancement of benign lesions. In addition, it can quantitatively characterize lesions by creating time-intensity curves and obtaining a series of quantitative parameters, including rise time, peak time (time to peak), peak intensity, mean transit time, and the AUC under the ROC curve.

The present study found that most fibroadenomas and intraductal papillomas manifested with true capsules or pseudocapsules caused by expansive growth; accordingly, if there was high enhancement on CEUS, the boundary was clear and the enhancement was well distributed. Malignant lesions of the breast, on the other hand, often lacked a capsule, and the boundary and size of the tumor, as well as the shape of nutrient angiography, could only be seen after enhancement. Since angiogenesis is a risk factor for invasion and metastasis of solid tumors, there are generally many proliferative and active cells at the perimeter of malignant tumors, as well as numerous abnormal capillary networks with disordered structures and increased microvessel densities. At the same time, perfusion disruptions can lead to increased microcirculation flow and velocity [19]. In addition, the peripheral regions of malignant breast tumors are often associated with breast hyperplasia and precancerous lesions at different stages. With the progression of these precancerous lesions to breast cancer, neovascularization and blood vessel density also increase [20].

In this study, adenoses generally manifested without pseudocapsules and exhibited an irregular shape after enhancement, which was often low or equally distributed, with a focus that either remained unchanged, became narrowed, or was completely integrated with the surrounding glands after enhancement. Further, few nutrient vessels and filling defects were manifested. These features can be helpful for differential diagnosis; however, some special adenoses exhibit uneven enhancement and unclear boundaries and can, therefore, easily be misdiagnosed as malignant nodules. Some inflammatory lesions also showed malignant signs after enhancement, including uneven high enhancement, irregular shapes, and unclear boundaries, which likely resulted from irregular infiltration of inflammatory cells into the surrounding tissue.

Real-time SWE can indicate differences in tissue hardness by measuring the propagation velocity of shear waves in tissues and can also qualitatively and quantitatively distinguish benign from malignant lesions. The elastic coefficient, or hardness, of the tissue is closely associated with the biological characteristics of lesions. The stroma of benign tumors, such as breast fibroadenomas, are rich in loose mucopolysaccharides; thus, their hardness is reduced in comparison to malignant tumors like invasive ductal carcinomas, in which the stroma is denser and harder due to fibrous tissue components [21]. In general, tumors are softer if they have a higher proportion of parenchyma than stroma. Tumor tissues can also soften when undergoing necrosis and harden when calcareous deposition or bone formation occurs. Real-time SWE is a relatively easy, noninvasive, and objective method to evaluate tissue hardness, and this study, as well as others, has demonstrated that it can effectively be used to differentiate benign from malignant breast tumors. 
Consistent with our previous study, we found that SWE had a high diagnostic sensitivity and specificity for BI-RADS category 4 nodules and small breast tumors. These findings are also consistent with other studies, both based in China and elsewhere [22,23]. Many studies have shown that tissue density information obtained by SWE can predict the degree of vascular infiltration, which is a predictor of lymph node metastasis $[24,25]$. Tumor cells can infiltrate the surrounding stroma and cause changes to connective tissue, increasing collagen cross-linking and the corresponding density of surrounding tissues. Using color gradients, SWE can demonstrate the hardness of areas surrounding lesions, with high-density areas displaying "hard ring signs" [26]. Unfortunately, this technique can also lead to misdiagnosis, as some malignant lesions undergo liquefactive necrosis, which results in decreased SWV values and falsenegative diagnoses. The SWV values for intraductal carcinomas with low tumor cell atypia, reduced proliferation of fibrous tissue, and no obvious infiltration into surrounding tissues have also been shown to be below the cut-off value. Some sclerosing benign breast lesions may also lead to an increase in the elastic modulus of tissues, resulting in false-positive diagnoses.

In our study, the combined use of CEUS, SWE, and BI-RADS led to adjustments in the BI-RADS classification for some breast nodules, with improvements in the diagnostic accuracies for benign and malignant breast nodules. Sclerosing adenoses were most commonly identified to be false positives, with some cases showing irregular morphologies due to interstitial fiber hyperplasia that was sometimes mixed with inflammation. In these instances, the SWV was higher than the cut-off value and CEUS showed uneven regions of high contrast enhancement. The malignant features of small breast carcinomas identified as false negatives were not as obvious on two-dimensional ultrasonography, showing only large lobulation, homogeneous echo patterns, and no changes or enhancement in posterior features. The ultrasound images resembled those of benign tumors, with SWE values lower than the cutoff value and CEUS showing only uniformly low contrast enhancement. Therefore, it is necessary to comprehensively analyze many images for nodules with these features, with close follow-up recommended. In addition, patients who are less than 60 years of age with a higher risk of malignancy due to family history should be followed closely, and further puncture biopsies should be carried out if necessary.

The BI-RADS classification system is not without flaws, especially in patients with category 4 nodules (with a malignant probability greater than $2 \%$ but less than 95\%) [27]. In the United States, most BI-RADS category 4 lesions $(69 \%-95 \%)$ undergo puncture biopsies, even though only $22 \%$ to $33 \%$ of these lesions are malignant. A meta-analysis of studies from Europe and the United States showed that the overdiagnosis rate of breast cancer lesions using molybdenum target mammography was $52 \%$ with the current BI-RADS classification system [26]. In the present study, 120 nodules were initially evaluated as BIRADS category 4A, with 67 cases adjusted to BI-RADS category 3 after consideration of the CEUS and SWE results. This finding indicates that $55.8 \%(67 / 120)$ of BI-RADS 4A nodules would have undergone unnecessary puncture biopsies after conventional ultrasound if not combined with the CEUS and SWE results. 
The current study had several limitations. First, as a retrospective study, the potential bias in the selection of the participants could not be eliminated. Second, the sample size was small; therefore, studies on larger datasets with long-term follow-up are required.

\section{Conclusions}

In summary, the BI-RADS classification system in combination with CEUS and SWE techniques exhibited a high diagnostic efficacy and applicability for differentiating benign from malignant small breast masses. Furthermore, this combined diagnostic method optimized the classification of BI-RADS category $4 \mathrm{~A}$ masses, though further research related to this topic is warranted.

\section{Abbreviations}

ACC, accuracy; AUC, area under the curve; BI-RADS, Breast Imaging Reporting and Data System; CEUS, contrast-enhanced ultrasound; $\mathrm{Cl}$, confidence interval; MTT, mean transit time; NPV, negative predictive value; PPV, positive predictive value; RT, rise time; SEN, sensitivity; SPE, specificity; SWE, shear-wave elastography; SWV, shear wave velocity; TIC, time-intensity curves; TTP, time to peak time

\section{Declarations}

Ethics approval and consent to participate: The protocol was approved by the Ethics Committee of Gong Li Hospital. The need for informed patient consent was waived due to the retrospective nature of this study.

Consent for publication: Not applicable

Availability of data and materials. The datasets used and/or analyzed during the current study are available from the corresponding authors on reasonable request.

Competing interests: The authors declare that they have no competing interests

Funding: None

Authors' Contributions: (I) Conception and design: YS and XHF; (II) Administrative support: YS and XHF; (III) Provision of study materials or patients: YS, XHF, JND; (IV) Collection and assembly of data: JH, ML, JJH, YLW, TTZ, JD, JND; (V) Data analysis and interpretation: YS and JH; (VI) Manuscript writing: YS, JH, JND, XHF; (VII) Final approval of manuscript: All authors

Acknowledgments: Not applicable 
Open Access Statement: This is an Open Access article distributed in accordance with the Creative Commons Attribution-NonCommercial-NoDerivs 4.0 International License (CC BY-NC-ND 4.0), which permits the non-commercial replication and distribution of the article with the strict proviso that no changes or edits are made and the original work is properly cited (including links to both the formal publication through the relevant DOI and the license). See: https://creativecommons.org/licenses/by-nc$\mathrm{nd} / 4.0 \%$.

\section{References}

1. Siegel RL, Miller KD, Jemal A. Cancer statistics, 2020. CA Cancer J Clin. 2020;70(1):7-30.

2. DeSantis CE, Ma J, Goding Sauer A, Newman LA, Jemal A. Breast cancer statistics, 2017, racial disparity in mortality by state. CA Cancer J Clin. $2017 ; 67(6): 439-48$.

3. Zhu YC, Zhang Y, Deng SH, Jiang Q. Diagnostic Performance of Superb Microvascular Imaging (SMI) Combined with Shear-Wave Elastography in Evaluating Breast Lesions. Med Sci Monit. 2018 ;24:5935-42.

4. PISTOLESE CA, TOSTI D, CITRARO D, et al. Probably benign breast nodular lesions (BI-RADS 3): correlation between ultrasound features and histologic findings . Ultrasound Med Biol. 2019;45 (1): 78-84.

5. YEO SH, KIM GR, LEE SH, et al. Comparison of ultrasound elastography and color doppler ultrasonography for distinguishing small triple-negative breast cancer from fibroadenoma. $\mathrm{J}$ Ultrasound Med.2018;37(9): 2135-46.

6. Mercado CL. BI-RADS update. Radiol Clin North Am. 2014 ;52(3):481-7.

7. Ji CL, Li XL, He YP, Li DD, Gu XG, Xu HX. Quantitative parameters of contrast-enhanced ultrasound in breast invasive ductal carcinoma: The correlation with pathological prognostic factors. Clin Hemorheol Microcirc. 2017;66(4):333-45.

8. Liu G, Zhang MK, He Y et al.BI-RADS 4 breast lesions: could multi-mode ultrasound be helpful for their diagnosis?.Gland Surg.2019;8(3):258-70.

9. Xiang $\mathrm{LH}, \mathrm{Yao} \mathrm{MH}, \mathrm{Xu} \mathrm{G}$ et al. Diagnostic value of contrast-enhanced ultrasound and shear-wave elastography for breast lesions of sub-centimeter. Clin Hemorheol Microcirc.2017; 67(1):69-80.

10. Luo J, Chen J D, Chen Q, et al. Application value of predictive models of contrast-enhanced ultrasound in the evaluation of breast imaging reporting and data system 4 breast lesions. Chin $\mathrm{J}$ Med Ultrasound.2016;13(6):459-65.

11. Nothacker M, Duda V, Hahn M, Warm M, Degenhardt F, Madjar H, Weinbrenner S, Albert US. Early detection of breast cancer: benefits and risks of supplemental breast ultrasound in asymptomatic women with mammographically dense breast tissue. A systematic review. BMC Cancer. 2009;9:335.

12. Lee CH, Dershaw DD, Kopans D, Evans P, Monsees B, Monticciolo D, Brenner RJ, Bassett L, Berg W, Feig $S$, et al. Breast cancer screening with imaging: recommendations from the Society of Breast 
Imaging and the ACR on the use of mammography, breast MRI, breast ultrasound, and other technologies for the detection of clinically occult breast cancer. J Am Coll Radiol. 2010;7(1):18-27.

13. Nam SY, Ko EY, Han BK, Shin JH, Ko ES, Hahn SY. Breast Imaging Reporting and Data System Category 3 Lesions Detected on Whole-Breast Screening Ultrasound. J Breast Cancer. 2016 ;19(3):301-7.

14. Castro SM, Tseytlin E, Medvedeva O, Mitchell K, Visweswaran S, Bekhuis T, Jacobson RS. Automated annotation and classification of BI-RADS assessment from radiology reports. J Biomed Inform. 2017;69:177-87.

15. Wan C, Du J, Fang H, Li F, Wang L. Evaluation of breast lesions by contrast enhanced ultrasound: qualitative and quantitative analysis. Eur J Radiol. 2012;81(4):e444-50.

16. Schroeder RJ, Bostanjoglo M, Rademaker J, Maeurer J, Felix R. Role of power Doppler techniques and ultrasound contrast enhancement in the differential diagnosis of focal breast lesions. Eur Radiol. 2003;13(1):68-79.

17. Athanasiou A, Tardivon A, Tanter M, Sigal-Zafrani B, Bercoff J, Deffieux T, Gennisson JL, Fink M, Neuenschwander S. Breast lesions: quantitative elastography with supersonic shear imagingpreliminary results. Radiology. 2010;256(1):297-303.

18. Youk JH, Gweon HM, Son EJ. Shear-wave elastography in breast ultrasonography: the state of the art. Ultrasonography. 2017;36(4):300-9.

19. Liu J, Gao YH, Li DD, Gao YC, Hou LM, Xie T. Comparative study of contrast-enhanced ultrasound qualitative and quantitative analysis for identifying benign and malignant breast tumor lumps. Asian Pac J Cancer Prev. 2014;15(19):8149-53.

20. Jiang YX, Liu H, Liu JB, Zhu QL, Sun Q, Chang XY. Breast tumor size assessment: comparison of conventional ultrasound and contrast-enhanced ultrasound. Ultrasound Med Biol. 2007 ;33(12):187381.

21. Aouad P, Saikali M, Abdel-Samad R, Fostok S, El-Houjeiri L, Pisano C, Talhouk R, Darwiche N. Antitumor activities of the synthetic retinoid ST1926 in two-dimensional and three-dimensional human breast cancer models. Anticancer Drugs. 2017;28(7):757-70.

22. Park J, Woo OH, Shin HS, Cho KR, Seo BK, Kang EY. Diagnostic performance and color overlay pattern in shear wave elastography (SWE) for palpable breast mass. Eur J Radiol. 2015;84(10):19438.

23. Ko ES, Lee BH, Kim HA, Noh WC, Kim MS, Lee SA. Triple-negative breast cancer: correlation between imaging and pathological findings. Eur Radiol. 2010;20(5):1111-7.

24. Wojcinski S, Soliman AA, Schmidt J, Makowski L, Degenhardt F, Hillemanns P. Sonographic features of triple-negative and non-triple-negative breast cancer. J Ultrasound Med. 2012;31(10):1531-41.

25. Çelebi F, Pilancı KN, Ordu Ç, Ağacayak F, Alço G, Illgün S, Sarsenov D, Erdoğan Z, Özmen V. The role of ultrasonographic findings to predict molecular subtype, histologic grade, and hormone receptor status of breast cancer. Diagn Interv Radiol. 2015;21(6):448-53. 
26. Berg WA, Zhang Z, Lehrer D, Jong RA, Pisano ED, Barr RG, Böhm-Vélez M, Mahoney MC, Evans WP 3rd, Larsen LH, et al. Detection of breast cancer with addition of annual screening ultrasound or a single screening MRI to mammography in women with elevated breast cancer risk. JAMA. 2012;307(13):1394-404.

27. Jørgensen KJ, Gøtzsche PC. Overdiagnosis in publicly organised mammography screening programmes: systematic review of incidence trends. Version 2. BMJ. 2009 9;339: b2587.

\section{Tables}

TABLE 1 Summary of demographic and ultrasonographic features

\begin{tabular}{|c|c|c|c|c|c|}
\hline \multirow[t]{2}{*}{ Parameter } & \multicolumn{2}{|c|}{ Pathological result } & \multirow[t]{2}{*}{ Total } & \multirow[t]{2}{*}{$t / \chi^{2}$} & \multirow[t]{2}{*}{$P$-value } \\
\hline & Benign & Malignant & & & \\
\hline No. of nodules & $\mathrm{n}=192$ & $\mathrm{n}=113$ & $\mathrm{n}=305$ & & \\
\hline Age, years & & & & 0.693 & 0.406 \\
\hline Mean & $42 \pm 14$ & $60 \pm 12$ & & & \\
\hline Range & $18-83$ & $36-84$ & & & \\
\hline Tumor size (mm) & & & & 2.452 & 0.118 \\
\hline Mean & $13.8 \pm 4.3$ & $14.5 \pm 3.8$ & & & \\
\hline Range & $4-20$ & $5-20$ & & & \\
\hline Location & & & & 0.759 & 0.226 \\
\hline Right breast & $88(45.8)$ & $46(40.7)$ & 134 & & \\
\hline Left breast & $104(54.2)$ & $67(59.3)$ & 173 & & \\
\hline Shape & & & & 57.455 & $<0.001$ \\
\hline Oval & $106(55.2)$ & $13(11.5)$ & 119 & & \\
\hline Round & $8(4.2)$ & $7(6.2)$ & 15 & & \\
\hline Irregular & $78(40.6)$ & $93(82.3)$ & 171 & & \\
\hline Orientation & & & & 59.354 & $<0.001$ \\
\hline Parallel & $159(82.8)$ & $45(39.8)$ & 204 & & \\
\hline Non-parallel & $33(17.2)$ & $68(60.2)$ & 101 & & \\
\hline Margin & & & & 91.450 & $<0.001$ \\
\hline Circumscribed & $141(73.4)$ & 19(16.8) & 160 & & \\
\hline Not circumscribed & $51(26.6)$ & $94(83.2)$ & 145 & & \\
\hline Echo pattern & & & & 3.093 & 0.542 \\
\hline Hypoechoic & $169(88.0)$ & $101(89.4)$ & 270 & & \\
\hline Heterogeneous & $7(3.6)$ & $5(4.4)$ & 12 & & \\
\hline Complex cystic and solid & $15(7.8)$ & $7(6.2)$ & 22 & & \\
\hline Hyperechoic & $1(0.5)$ & $0(0.0)$ & 1 & & \\
\hline Posterior acoustic features & & & & 16.015 & 0.001 \\
\hline No. of posterior acoustic features & $172(89.6)$ & $94(83.2)$ & 266 & & \\
\hline Enhancement & $10(5.2)$ & $1(0.9)$ & 11 & & \\
\hline Shadowing & $7(3.6)$ & $17(15.0)$ & 24 & & \\
\hline Combined pattern & $3(1.6)$ & $1(0.9)$ & 4 & & \\
\hline Calcification & & & & 43.308 & $<0.001$ \\
\hline None & $156(81.3)$ & $54(47.8)$ & & & \\
\hline Calcification inside a mass & $35(18.2)$ & $52(46.0)$ & & & \\
\hline Calcification outside a mass & $1(0.5)$ & $0(0.0)$ & & & \\
\hline Intraductal calcification & $0(0.0)$ & $7(6.2)$ & & & \\
\hline Vascularity & & & & 35.871 & $<0.001$ \\
\hline Absent & $136(70.8)$ & $44(38.9)$ & & & \\
\hline Internal vascularity & $43(22.4)$ & $62(54.9)$ & & & \\
\hline Vessels in rim & $11(5.7)$ & $4(3.5)$ & & & \\
\hline Internal+vessels in rim & $2(1.0)$ & $3(2.7)$ & & & \\
\hline Lymph node metastasis & & & & 60.745 & $<0.001$ \\
\hline Normal & $192(100.0)$ & $79(69.9)$ & 271 & & \\
\hline Metastasis & $1(0.0)$ & $34(30.1)$ & 35 & & \\
\hline
\end{tabular}

Data in parentheses are percentages. 
Table 2

Diagnosis of benign and malignant nodules using four evaluation methods and pathology

\begin{tabular}{|c|c|c|c|c|c|}
\hline \multirow[t]{2}{*}{ Category } & \multicolumn{2}{|c|}{ Pathologic diagnosis } & \multirow[t]{2}{*}{ Total } & \multirow{2}{*}{$x^{2}$} & \multirow[t]{2}{*}{$P$-value } \\
\hline & Benign & Malignant & & & \\
\hline No. of nodules & $\mathrm{n}=192$ & $n=113$ & $\mathrm{n}=305$ & & \\
\hline BI-RADS & & & & 92.171 & $<0.001$ \\
\hline Benign & 143 & 20 & 163 & & \\
\hline Malignant & 49 & 93 & 142 & & \\
\hline CEUS & & & & 149.838 & $<0.001$ \\
\hline Benign & 168 & 19 & 187 & & \\
\hline Malignant & 24 & 94 & 118 & & \\
\hline SWE & & & & 143.380 & $<0.001$ \\
\hline Benign & 159 & 15 & 174 & & \\
\hline Malignant & 33 & 98 & 131 & & \\
\hline Combination & & & & 167.533 & $<0.001$ \\
\hline Benign & 167 & 13 & 180 & & \\
\hline Malignant & 25 & 100 & 125 & & \\
\hline \multicolumn{6}{|c|}{$\begin{array}{l}\text { Abbreviations: BI-RADS, Breast Imaging Reporting and Data System; CEUS, contrast-enhanced } \\
\text { ultrasound; SWE, shear-wave elastography }\end{array}$} \\
\hline
\end{tabular}

Table 3

Diagnostic efficacy of four examination methods

\begin{tabular}{|llllllll|}
\hline Parameter & SEN (\%) & SPE (\%) & PPV (\%) & NPV (\%) & ACC (\%) & AUC (\%) & 95\% Cl (\%) \\
\hline BI-RADS & 82.3 & 74.5 & 65.5 & 87.7 & 77.4 & 78.4 & $73.3-82.9$ \\
\hline CEUS & 83.2 & 87.5 & 79.7 & 89.8 & 85.9 & 85.3 & $80.9-89.1$ \\
\hline SWE & 86.7 & 82.8 & 74.8 & 91.4 & 84.3 & 84.8 & $80.2-88.6$ \\
\hline Combination & 88.5 & 87.0 & 80.0 & 92.8 & 87.5 & 87.7 & $83.5-91.2$ \\
\hline $\begin{array}{l}\text { Abbreviations: BI-RADS, Breast Imaging Reporting and Data System; CEUS, contrast-enhanced } \\
\text { ultrasound; SWE, shear-wave elastography; SEN, sensitivity; SPE, specificity; PPV, positive predictive } \\
\text { value; NPV, negative predictive value; ACC, accuracy; AUC, area under the curve; Cl, confidence interval }\end{array}$ \\
\hline
\end{tabular}

\section{Figures}




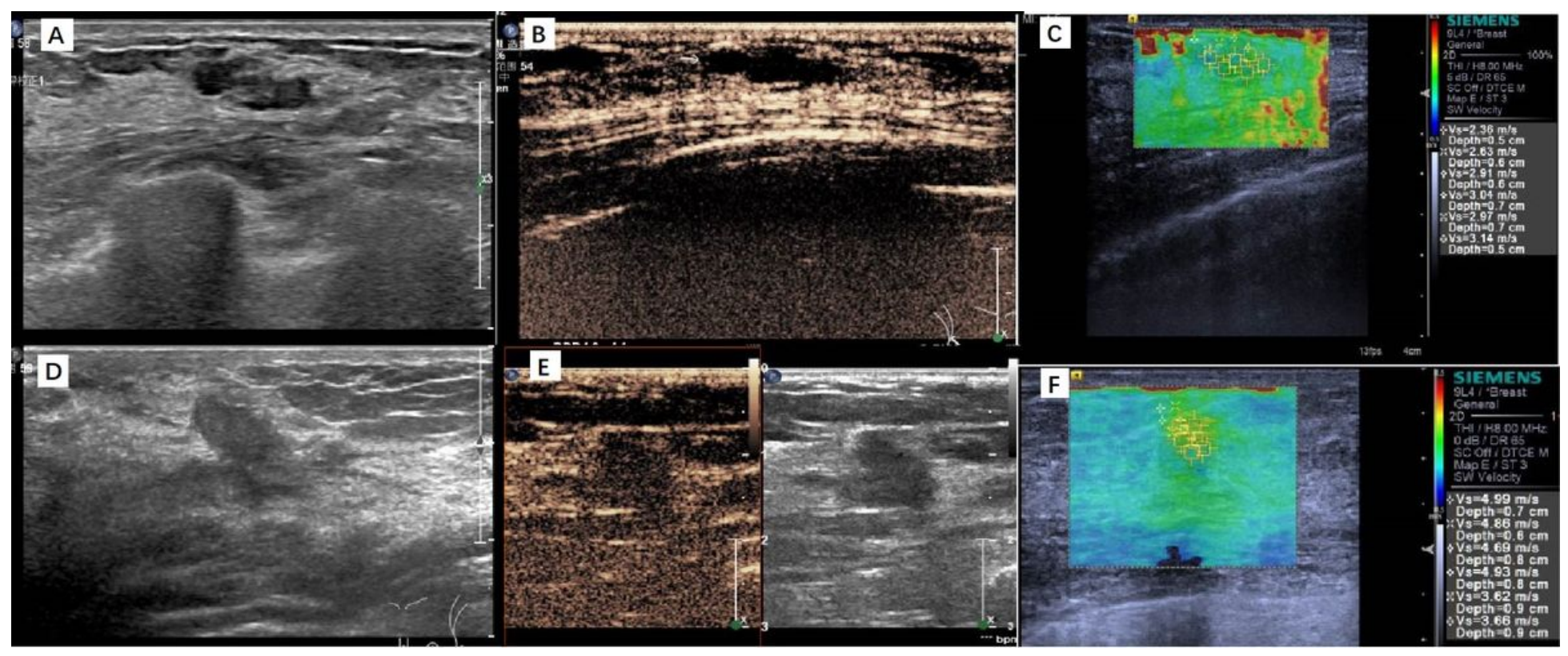

Figure 1

(A) The nodule is irregular and not circumscribed. BI-RADS is classified as 4A nodules. (B) CEUS showed no significant enhancement of nodules, and the volume decreased after enhancement. (C) The average SWV of SWE is $2.8 \mathrm{~m} / \mathrm{s}$, which is less than the cutoff value, indicating that the texture of the nodules is soft. After BI-RADS combined CEUS and SWE, BI-RADS was downgraded to BI-RADS 3. The final pathological result is breast adenopathy. (D) The nodule is not circumscribed and not parallel. BI-RADS is classified as 4A nodules. (E) CEUS showed unevenness and centripetal enhancement, and the volume increased after enhancement. (F) The average SWV of SWE is $4.1 \mathrm{~m} / \mathrm{s}$, which is more than the cutoff value, indicating that the nodules is hard. After BI-RADS combined CEUS and SWE, BI-RADS was downgraded to BI-RADS 4B. The final pathological result is Ductal carcinoma in situ. 


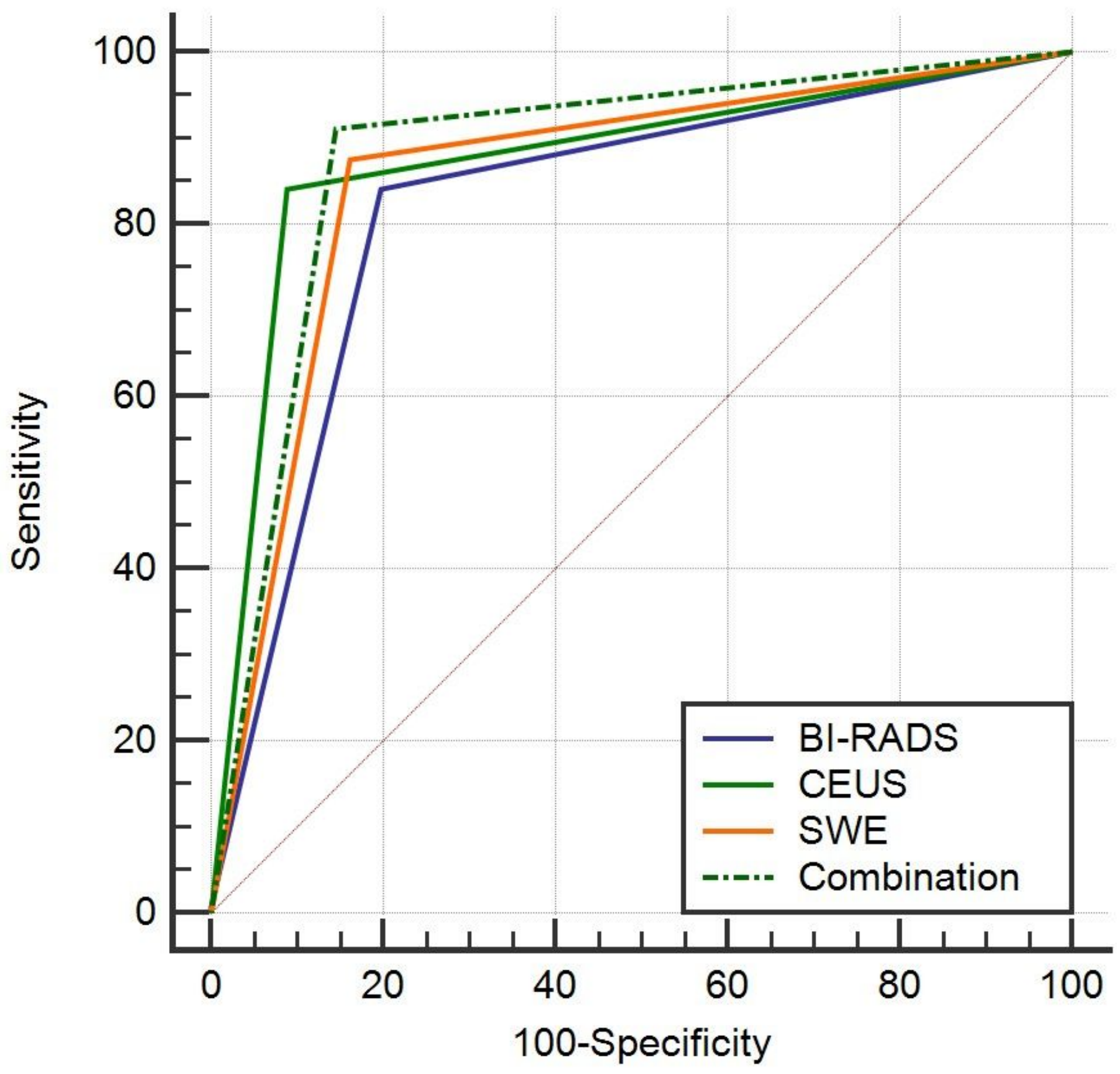

Figure 2

Analysis of the receiver operating characteristic curves of the four methods

\section{Supplementary Files}

This is a list of supplementary files associated with this preprint. Click to download.

- consortchecklist.doc 